\title{
Focusing of Azimuthally Polarized Hyperbolic-Cosine-Gaussian Beam
}

\author{
Xiumin Gao ${ }^{1,2}$, Mingyu Gao ${ }^{1}$, Song $\mathrm{Hu}^{1}$, Hanming $\mathrm{Guo}^{2}$, Jian Wang ${ }^{1}$, Songlin Zhuang ${ }^{2}$ \\ ${ }^{1}$ Electronics \& Information College, Hangzhou Dianzi University, Hangzhou, China \\ ${ }^{2}$ University of Shanghai for Science and Technology, Shanghai, China \\ E-mail:xiumin_gao@yahoo.com.cn,optics.hangzhou@gmail.com,husong1971@hotmail.com \\ Received October 3, 2009; revised November 2, 2009; accepted November 8, 2009
}

\begin{abstract}
The focusing properties of azimuthally polarized hyperbolic-cosine-Gaussian (ChG) beam are investigated theoretically by vector diffraction theory. Results show that the intensity distribution in focal region of azimuthally polarized $\mathrm{ChG}$ beam can be altered considerably by decentered parameters, and some novel focal patterns may occur for certain case. On increasing decentered parameters, ring shape of focal pattern can evolve into four-peak focal pattern, and azimuthal field component affects focal pattern more significantly than radial field component. Optical gradient force is also calculated to show that the focusing properties may be used in optical tweezers technique.
\end{abstract}

Keywords: Focusing Properties, Hyperbolic-Cosine-Gaussian Beam, Vector Diffraction Theory

\section{Introduction}

Laser beams with cylindrical symmetry in polarization have attracted many researchers recently for their interesting properties and applications. And these beams are called cylindrical vector beams and can be generated by active or passive methods [1-4]. Due to the polarization symmetry, the electric field at the focus has unique polarization properties. K. S. Youngworth and T. G. Brown calculated cylindrical-vector fields [2], which shows that, in the particular case of a tightly focused radially polarized beam, the polarization shows large inhomogeneities in the focal region, while the azimuthally polarized beam has purely transverse field in focal region. Focus shaping technique is also reported by using generalized cylindrical vector beams [3], in which a generalized cylindrical vector beam can be decomposed into radially polarized and azimuthally polarized components. And a generalized cylindrical beam can be generated from a radially polarized or an azimuthally polarized light using a two-halfwave-plate polarization rotator.

On the other hand, although Gaussian beam is familiar to researchers, it is only one kind of solution of the Helmholtz equation. The more general solution is Hermite-Sinusoidal-Gaussian beams, which was introduced by Casperson and coworkers [5,6]. Hyperbolic-cosineGaussian beams are regarded as the special case of Hermite-sinusoidal-Gaussian beams, and are of practical interest because their profiles can be altered by choosing suitable beam parameters in cosh parts [7]. Propagation and focusing properties of hyperbolic-cosineGaussian beams have become the object of some works [8-12]. To investigate focusing properties of this kind of light beam with azimuthally polarized distribution is very interesting, which may deepen understanding of its properties and expand application. In this paper, the focusing properties of the azimuthally polarized hyperbolic-cosine-Gaussian ( $\mathrm{ChG})$ beam are investigated theoretically by vector diffraction theory. In Section 2, the principle of the focusing system is given. And results and discussions are shown in Section 3. Conclusions are summarized in Section 4.

\section{Principle of the Focusing Azimuthally Polarized ChG Beam}

In the focusing system we investigated, focusing beam is azimuthally polarized $\mathrm{ChG}$ beam whose value of transverse optical field is same as that of the scalar ChG beam, and its polarization distribution turns on azimuthally symmetric. Therefore, in the cylindrical coordinate system $(r, \phi, 0)$ the field distribution $\vec{E}(r, \phi, 0)$ of the azimuthally polarized $\mathrm{ChG}$ beam is written as [1-3],

$$
\vec{E}_{0}(r, \varphi, 0)=E_{0}(r, \varphi, 0) \cdot \vec{n}
$$

where $\vec{n}$ is the azimuthal unit vector of polarized direction. Term $E_{0}(r, \varphi, 0)$ is optical field value distribu- 
tion and can be written in the form [7-9],

$$
E(x, y, 0)=B \cosh \left(\Omega_{x} x\right) \cosh \left(\Omega_{y} y\right) \exp \left(-\frac{x^{2}+y^{2}}{\omega_{0}^{2}}\right)
$$

where cosh is hyperbolic-cosine function, $\omega_{0}$ is the waist width of the beam. And $B$ is a constant. $\Omega_{x}$ and $\Omega_{y}$ indicate decentered parameters of ChG beam. According to vector diffraction theory, the electric field in focal region of azimuthally polarized $\mathrm{ChG}$ beam is [13],

$$
\vec{E}(\rho, \phi, z)=E_{\rho} \vec{e}_{\rho}+E_{\phi} \vec{e}_{\phi}
$$

where $\vec{e}_{\rho}$ and $\vec{e}_{\phi}$ are the unit vectors in the radial and azimuthal directions, respectively. To indicate the position in image space, cylindrical coordinates $(\rho, \phi, z)$ with origin $\rho=z=0$ located at the paraxial focus are employed. $E_{\rho}$ and $E_{\Phi}$ are the amplitudes of two orthogonal components and can be expressed as:

$$
\begin{gathered}
E_{\rho}(\rho, \phi, z)=\frac{i A}{\pi} \int_{0}^{\alpha} \int_{0}^{2 \pi} \sqrt{\cos \theta} \cdot E_{0} \cdot \sin \theta \sin (\varphi-\phi) \\
\cdot \exp \{i k[z \cos \theta+\rho \sin \theta \cos (\varphi-\phi)]\} d \varphi d \theta \\
E_{\phi}(\rho, \phi, z)=\frac{-i A}{\pi} \int_{0}^{\alpha} \int_{0}^{2 \pi} \sqrt{\cos \theta} \cdot E_{0} \cdot \sin \theta \cos (\varphi-\phi) \\
\cdot \exp \{i k[z \cos \theta+\rho \sin \theta \cos (\varphi-\phi)]\} d \varphi d \theta
\end{gathered}
$$

where $\theta$ and $\varphi$ denote the tangential angle with respect to the $z$ axis and the azimuthal angle with respect to the $x$ axis, respectively. $k$ is wave number. $\alpha=\arcsin (N A)$ is convergence angle corresponding to the radius of incident optical aperture. In order to make focusing properties clear and simplify calculation process, after simple derivation, Equation 2 can be rewritten as [11]:

$$
\begin{aligned}
& E(\theta, \varphi)=B \cdot \cosh \left[N A^{-1} \cdot \beta_{x} \cdot \sin (\theta) \cdot \cos (\varphi)\right] \\
& \cdot \cosh \left[N A^{-1} \cdot \beta_{y} \cdot \sin (\theta) \cdot \sin (\varphi)\right] \\
& \cdot \exp \left[-\frac{\sin ^{2}(\theta)}{N A^{2} \cdot w^{2}}\right]
\end{aligned}
$$

where $w=\omega_{0} / r_{0}$ is called relative waist width. Parameters $\beta_{x}=r_{p} \Omega_{x}$ and $\beta_{y}=r_{p} \Omega_{y}$ in this paper are called decentered parameters of azimuthally polarized $\mathrm{ChG}$ beam. Substitute the Equation 6 into Equations 4 and 5, we can obtain:

$$
\begin{aligned}
& E_{\rho}(\rho, \phi, z)=\frac{i A B}{\pi} \int_{0}^{\alpha} \int_{0}^{2 \pi} \sqrt{\cos \theta} \sin \theta \sin (\varphi-\phi) \\
& \cdot \cosh \left[N A^{-1} \cdot \beta_{x} \cdot \sin (\theta) \cdot \cos (\varphi)\right] \cdot \exp (i \psi) \\
& \cdot \cosh \left[N A^{-1} \beta_{y} \sin (\theta) \sin (\varphi)\right] \cdot \exp \left[-\frac{\sin ^{2}(\theta)}{N A^{2} \cdot w^{2}}\right]
\end{aligned}
$$

$$
\begin{aligned}
& \cdot \exp \{i k[z \cos \theta+\rho \sin \theta \cos (\varphi-\phi)]\} d \varphi d \theta \\
& E_{\phi}(\rho, \phi, z)=\frac{-i A B}{\pi} \int_{0}^{\alpha} \int_{0}^{2 \pi} \sqrt{\cos \theta} \sin \theta \cos (\varphi-\phi) \\
& \cdot \cosh \left[N A^{-1} \cdot \beta_{x} \cdot \sin (\theta) \cdot \cos (\varphi)\right] \cdot \exp (i \psi) \\
& \cdot \cosh \left[N A^{-1} \beta_{y} \sin (\theta) \sin (\varphi)\right] \cdot \exp \left[-\frac{\sin ^{2}(\theta)}{N A^{2} \cdot w^{2}}\right] \\
& \cdot \exp \{i k[z \cos \theta+\rho \sin \theta \cos (\varphi-\phi)]\} d \varphi d \theta
\end{aligned}
$$

The optical intensity in focal region is proportional to the modulus square of Equation 3. Based on the above equations, focusing properties of azimuthally polarized $\mathrm{ChG}$ beam can be investigated in detail.

\section{Results and Discussions}

Without loss of validity and generality, it was supposed that $N A=0.95$ and $w=1$. Firstly, the intensity distributions in focal region of azimuthally polarized $\mathrm{ChG}$ beam under condition of different decentered parameters are calculated and illustrated in Figure 1. It should be noted that $\beta=\beta_{x}=\beta_{y}$, and the distance unit in all figures in this paper is $\mathrm{k}^{-1}$, where $k$ is the wave number of incident beam. The azimuthal angle ranges from $-\pi$ to $\pi$ in azimuthal coordinate. It can be seen from Figure 1 that the intensity distribution turns on one ring shape in focal plane for small decentered parameter $\beta$, which is similar to that for Gaussian beam. On increasing $\beta$, the focal pattern changes very remarkably, from one ring focal pattern to four-peak focal pattern, and these four intensity peaks overlap very considerably, as shown in Figure 1(b). Increase decentered parameter $\beta$ continuously, there appear several weak overlapping intensity peaks outside of the center four-peak overlapping focal pattern, in addition, multiple dark focal spots occur between inner four-peak focal pattern and outer multiple overlapping intensity pattern. And dark focal spots get more obvious on increasing $\beta$, such as in Figure 1(f). From above focal pattern evolution, we can see that intensity distribution in focal region of azimuthally polarized ChG beam can be altered considerably by decentered parameters, and some novel focal patterns may occur.

In order to understand the focusing properties deeply, the different optical field components in focal region are also investigated. And one calculation example is given here. Figure 2 illustrates the radial field component for $\beta=2$ and $\beta=17$, and the corresponding azimuthal field component is given in Figure 3 .

The radial field component distribution turns multiple optical intensity peaks outside of optical axis, and changes slightly under condition of different $\beta$. While, azimuthal field component distribution changes very considerably on increasing $\beta$, evolving from one focal ring pattern to multiple overlapping optical intensity peaks in focal plane. 


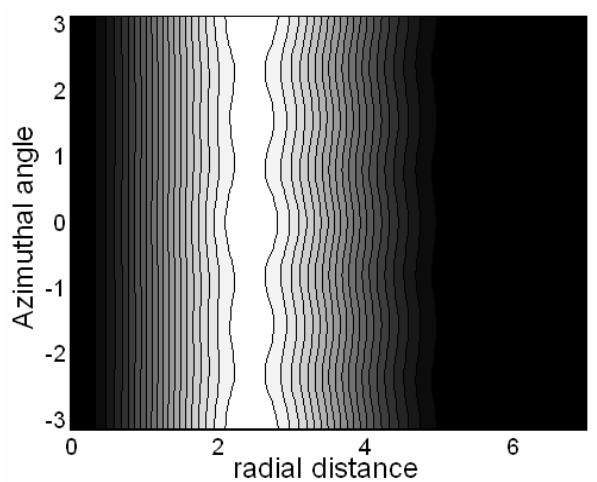

(a)

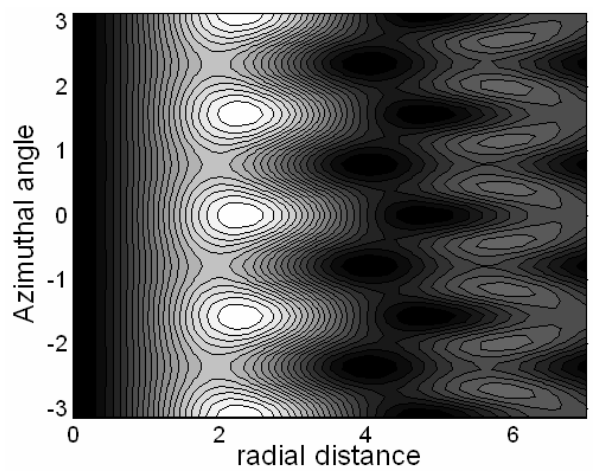

(c)

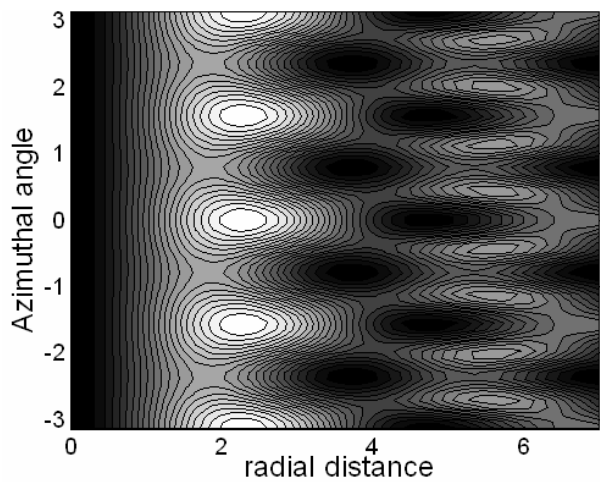

(e)

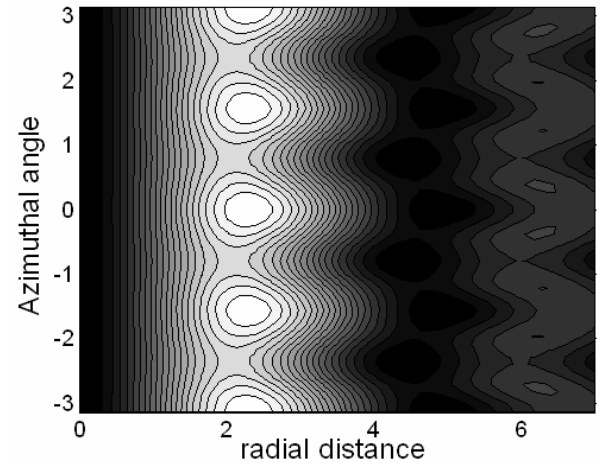

(b)

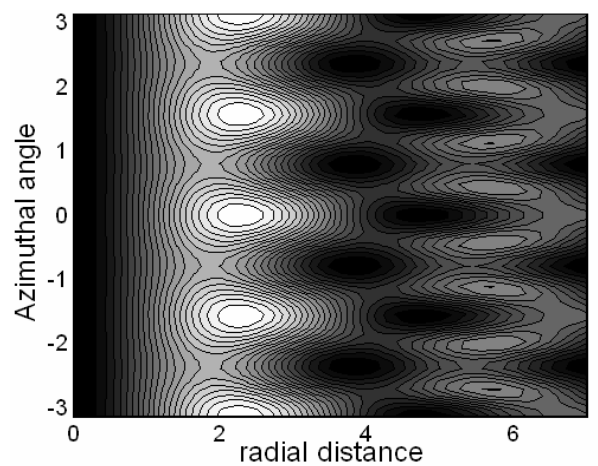

(d)

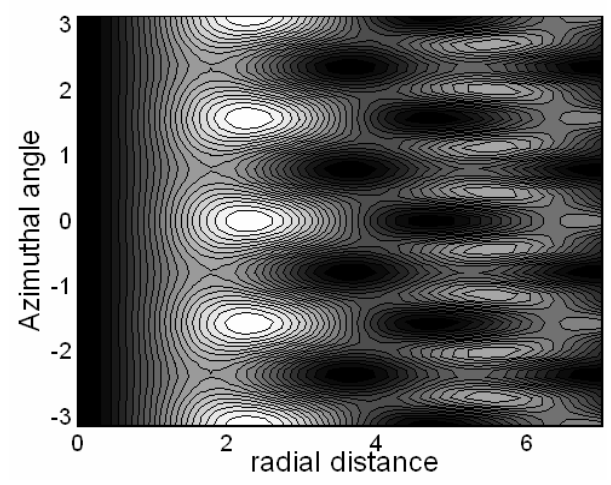

(f)

Figure 1. Intensity distributions in focal region for (a) $\beta=2$, (b) $\beta=5$, (c) $\beta=8$, (d) $\beta=11$, (e) $\beta=14$, and (f) $\beta=17$, respectively.

By comparing Figures 2 and 3 with Figure 1, it can be seen that azimuthal field component distribution is very similar to total focal pattern. Therefore, azimuthal field component affects focal pattern more significantly than radial field component, namely, azimuthal field component decides optical intensity distribution in focal region of the azimuthally polarized $\mathrm{ChG}$ beam.

Now, the possible application of focusing of azimuthally polarized $\mathrm{ChG}$ beam is discussed. Optical tweezers have been increasingly valuable tool for research and accelerated many major advances in numerous areas of science [14-17]. In optical tweezers system, it is usually deemed that the forces exerted on the particles in light field include two kinds of forces, one is the gradient force, which is proportional to the intensity gradient; the other is the scattering force, which is proportional to the optical intensity [16]. So, the tunable focal pattern predicts that the optical trap may be controllable. The gradient force trap is necessary condition for constructing the optical trap and can be expressed as [16]

$$
F_{\text {grad }}=\frac{n_{b}^{2} r^{3}}{2} \cdot\left(\frac{m^{2}-1}{m^{2}+2}\right) \nabla|\vec{E}(\rho, \phi, z)|^{2}
$$

where $r$ is the radius of trapped particles, $n_{b}$ is the refraction index of the surrounding medium, and $m$, the relative index of refraction, equals to the ratio of the refraction index of the particle $n_{p}$ to the refraction index of the 


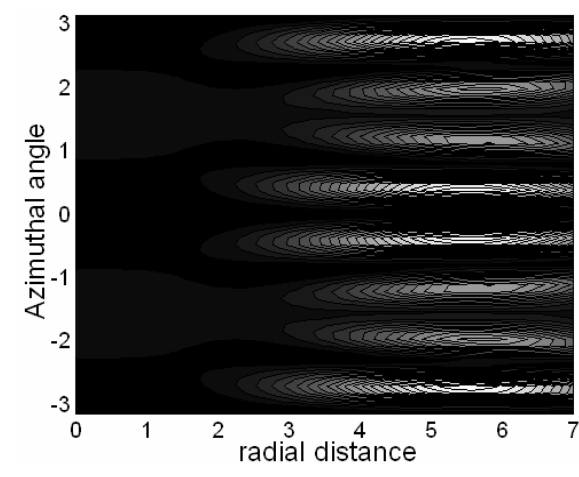

(a)

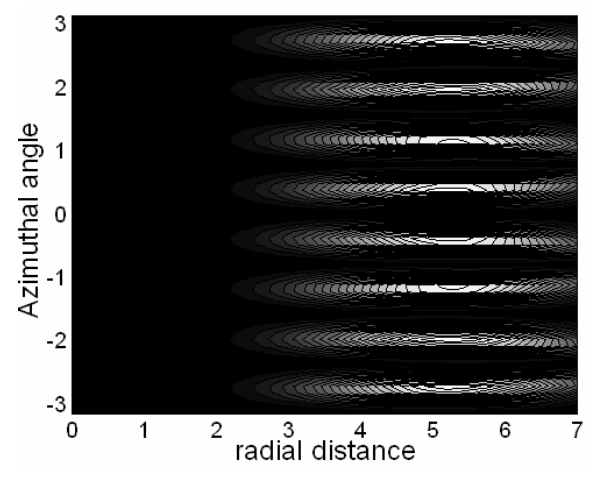

(b)

Figure 2. Radial field component for (a) $\beta=2$ and (b) $\beta=17$, respectively.

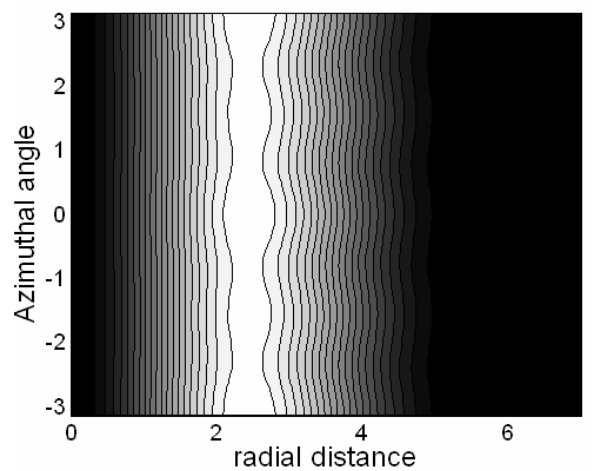

(a)

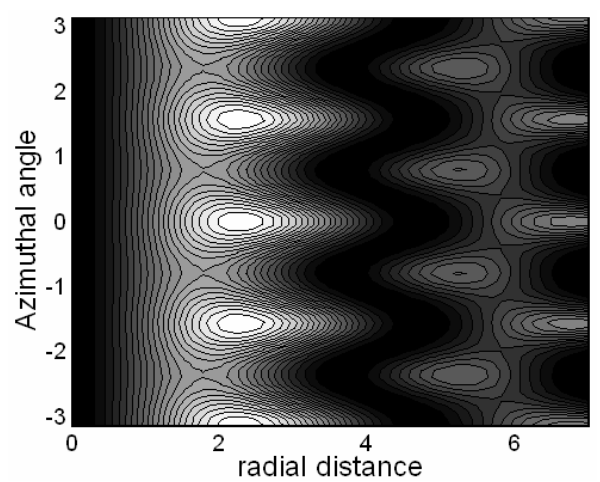

(b)

Figure 3. Azimuthal field component for (a) $\beta=2$ and (b) $\beta=17$, respectively.

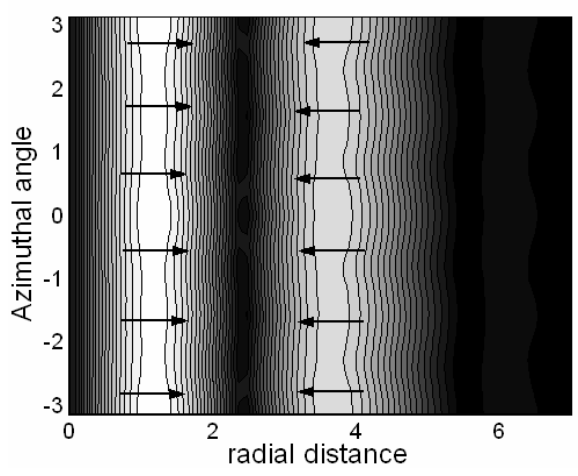

(a)

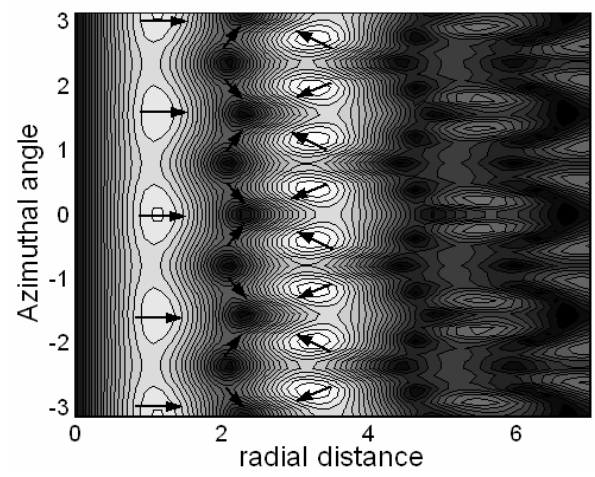

(b)

Figure 4. Optical gradient force for (a) $\beta=2$ and (b) $\beta=5$, respectively. The arrows indicate the direction of optical gradient force in focal region.

surrounding medium $n_{b}$. Gradient force $F_{\text {grad }}$ points in the direction of the gradient of the light intensity for $n_{p}>n_{b}$. The optical gradient force can be computed numerically by substituting Equation 3 into Equation 9. Figure 4 illustrates the optical gradient force for $\beta=2$ and $\beta=5$. The arrows indicate the direction of optical gradient force in focal region. There is one ring optical trap for $\beta=2$, and multiple optical traps under condition of $\beta=5$. Decentered parameters can change focal pattern very remarkable. Therefore, the focusing properties of azimuthally polarized $\mathrm{ChG}$ beam can be constructed to tunable optical traps.

\section{Conclusions}

The focusing properties of azimuthally polarized $\mathrm{ChG}$ beam are investigated theoretically by vector diffraction theory. Simulations results show that the intensity distribution in focal region of azimuthally polarized $\mathrm{ChG}$ beam can be altered considerably by decentered parameters and some novel focal patterns may occur. For instance, ring focal pattern, four-peak focal pattern, and dark focal spot. On increasing decentered parameters, ring shape of focal pattern can evolve into four-peak fo- 
cal pattern, which may be used to construct tunable optical tweezers. In addition, azimuthal field component affects focal pattern more significantly than radial field component.

\section{Acknowledgments}

This work was supported by National Basic Research Program of China (2005CB724304), National Natural Science Foundation of China $(60708002$, 60807007, 60871088, 60778022), China Postdoctoral Science Foundation (20080430086), Shanghai Postdoctoral Science Foundation of China (08R214141), and Shanghai Leading Academic Discipline Project (S30502).

\section{References}

[1] Q. Zhan, "Cylindrical vector beams: From mathematical concepts to applications," Advances in Optics and Photonics, Vol. 1, pp. 1-57, 2009.

[2] K. S. Youngworth and T. G. Brown, "Focusing of high numerical aperture cylindrical-vector beams," Optics Express, Vol. 7, pp. 77-87, 2000.

[3] X. Gao, J. Wang, H. Gu, and W. Xu, "Focusing properties of concentric piecewise cylindrical vector beam," Optik, Vol. 118, pp. 257-265, 2007.

[4] G. Zhou, Y. Ni, and Z. Zhang, "Analytical vectorial structure of non-paraxial nonsymmetrical vector Gaussian beam in the far field," Optics Communications, Vol. 272, pp. 32-39, 2007.

[5] L. W. Casperson, D. G. Hall, and A. A. Tovar, "SinusoidalGaussian beams in complex optical systems," Journal of Optical Society of America A, Vol. 14, pp. 3341-3348, 1997.

[6] L. W. Casperson and A. A. Tovar, "Hermite-sinusoidalGaussian beams in complex optical systems," Journal of
Optical Society of America A, Vol. 15, pp. 954-961, 1998.

[7] X. Du and D. Zhao, "Elliptical cosh-Gaussian beams," Optics Communications, Vol. 265, pp. 418-424, 2006.

[8] Z. Hricha and A. Belafhal, "Focusing properties and focal shift in hyperbolic-cosine-Gaussian beams," Optics Communications, Vol. 253, pp. 242-249, 2005.

[9] B. Lu and S. Luo, "Beam propagation factor of hard-edge diffracted cosh-Gaussian beams," Optics Communications, Vol. 178, pp. 275-281, 2000.

[10] B. Lu, H. Ma, and B. Zhang, "Propagation properties of cosh-Gaussian beams," Optics Communications, Vol. 164, pp. 165-170, 1999.

[11] X. Gao, "Focusing properties of the hyperbolic-cosineGaussian beam induced by phase plate," Physics Letters A, Vol. 360, pp. 330-335, 2006.

[12] X. Gao and J. Li, "Focal shift of apodized tunrcated hyperbolic-cosine-Gaussian beam," Optics Communications, Vol. 273, pp. 21-27, 2007.

[13] S. Sato and Y. Kozawa, "Hollow vortex beams," Journal of Optical Society of America A, Vol. 26, pp. 142-146, 2009.

[14] M. P. MacDonald, L. Paterson, K. V. Sepulveda, J. Arlt, W. Sibbett, and K. Dholakia, "Creation and manipulation of three-dimensional optically trapped structures," Science, Vol. 296, pp. 1101-1103, 2002.

[15] D. G. Grier, "A revolution in optical manipulation," Nature, Vol. 424, pp. 810-816, 2003.

[16] V. Garces-Chaves, D. McGloin, H. Melville, W. Sibbett, and K. Dholakia, "Simultaneous micromanipulation in multiple planes using a self reconstructing light beam," Nature, Vol. 419, pp. 145-147, 2002.

[17] K. Visscher and G. J. Brakenhoff, "Theoretical study of optically induced forces on spherical particles in a single beam trap I: Rayleigh scatterers," Optik, Vol. 89, pp. 174-180, 1992. 